Article

\title{
The Prediapause Stage of Aedes japonicus japonicus and the Evolution of Embryonic Diapause in Aedini
}

\author{
Jake Bova ${ }^{1, *}$, John Soghigian ${ }^{2}$ and Sally Paulson ${ }^{3}$ \\ 1 Department of Biology, Emory and Henry College, Emory, VA 24327, USA \\ 2 Department of Entomology and Plant Pathology, North Carolina State University, Raleigh, NC 27607, USA \\ 3 Department of Entomology, Virginia Tech, Blacksburg, VA 24061, USA \\ * Correspondence: jbova@ehc.edu; Tel.: +1-276-944-6775
}

Received: 4 June 2019; Accepted: 15 July 2019; Published: 25 July 2019

check for updates

\begin{abstract}
The genus Aedes is well known for its desiccation-resistant eggs, which frequently serve as an overwintering mechanism through diapause. Despite this, relatively little is known about the diapause and overwintering biology of most Aedes species including Aedes japonicus japonicus, an invasive mosquito in the United States. The importance of this mosquito in disease systems like La Crosse virus remain uncertain. Embryonic diapause is used by Ae. j. japonicus to survive temperate winters, and the persistence of this species in the Appalachian region is a result of overwintering, which has important implications for the transmission of this virus to humans. The objective of this study was to identify the prediapause stage, or the stage sensitive to environmental cues needed to induce diapause in this mosquito. By exposing each Ae. j. japonicus life stage independently to short-day photoperiods, we determined that the adult maternal life stage is the prediapause stage. Using the most recent phylogeny and prior literature on the prediapause stages in the genus Aedes, we were able to infer the evolutionary history of the prediapause stages of Aedes mosquitoes that overwinter or aestivate as eggs. This initial ancestral state reconstruction allowed us to hypothesize that Aedini mosquitoes that undergo obligate diapause may have evolved from those utilizing the embryonic prediapause stage, and that the ancestral prediapause state of Aedini appears to be maternally controlled.
\end{abstract}

Keywords: mosquito; diapause; overwinter; ancestral state reconstruction

\section{Introduction}

Many mosquitoes are faced with adverse weather conditions due to their geographic range, and a major survival strategy is to enter into a hibernal diapause to shield themselves from low temperature extremes. Multivoltine, temperate mosquitoes utilize a facultative diapause usually driven by shorter photoperiods and temperature. Most temperate Aedes mosquitoes, as well as those within the tribe they belong to, the Aedini, overwinter as the pharate first instar within their egg, but the life-cycle stage that is sensitive to environmental cues that induce diapause, referred to as the prediapause stage, can differ between Aedes species. Either the developing embryo or maternal generation is sensitive to light and temperature to induce embryonic diapause (Figure 1) [1-3]. 


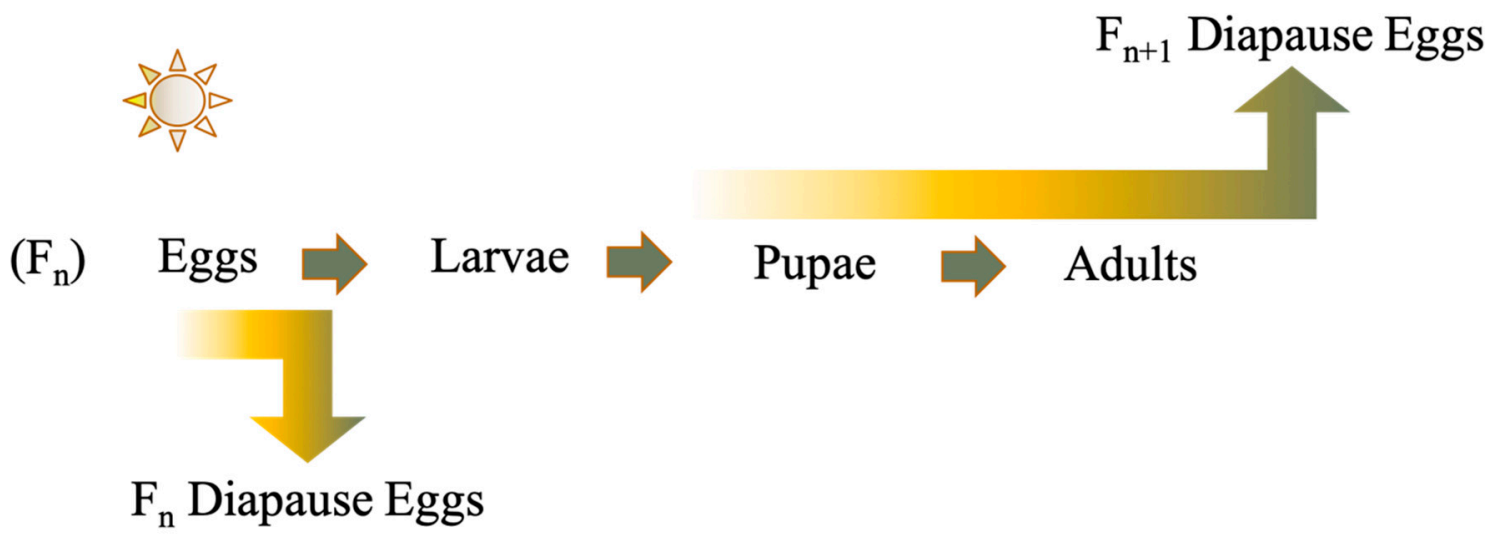

Figure 1. Two common photoperiodic diapause induction methods utilized by facultative embryonic diapause in the genus Aedes. Gradient arrows indicate the ability of the life-cycle stage to sense environmental cues needed to enter diapause either in the $F_{n}$ generation or $F_{n+1}$ generation.

Aedes japonicus japonicus is an invasive mosquito in the United States and is capable of vectoring several viruses [4]. This mosquito is a cold-adapted species and has been known to overwinter predominantly as eggs, but some overwintering larvae have been identified [5]. In the Appalachia region, La Crosse virus- and Cache Valley virus-infected pools of Ae. j. japonicus have been collected [6-8]. Its invasion biology is similar to that of the Asian tiger mosquito Aedes albopictus. While a common mosquito in the eastern United States, much remains to be discovered about Ae. j. japonicus's basic biology and its increasingly pertinent vector status makes this imperative to understanding future disease emergence related to this species.

The goal of this study was to identify the prediapause stage of Ae. j. japonicus and to better understand the evolution of the diapause system by mapping the known phylogeny for the prediapause stage of Aedes mosquitoes that undergo embryonic diapause. Previous discussion on the evolution of diapause has centered around the diapause stage [1]; however, we show here the prediapause phenotype should be considered when evaluating the evolution of embryonic diapause in Aedini.

\section{Materials and Methods}

The Ae. j. japonicus we used in this study were collected using modified Reiter gravid traps from a forested area in Montgomery County during the month of July [8]. Adult mosquitoes were allowed to oviposit upon seed germination paper and were then screened for possible viral infection by plaque assay. All mosquitoes were virus-negative.

To elucidate the prediapause stage of Ae. j. japonicus, populations of a single life stage of the $\mathrm{F}_{2}$ generation were exposed to a 8:16 (L:D) short-day photoperiod, while the remainder $\mathrm{F}_{2}$ life stages were exposed to a 16:8 (L:D) long-day photoperiod according to modified methods from Kappus and Venard [9]. As such, the egg, larval, pupal, and adult life stages were all exclusively exposed to the short-day photoperiod, while the additional life stages from the same cohort were exposed to long-day photoperiods, e.g., for mosquitoes treated with short-day photoperiods as larvae, eggs, pupae, and adults of this cohort were exposed to long-day photoperiods. All $F_{1}$ mosquitoes were exposed to long-day photoperiods, including the cohort where eggs were subjected to short-day photoperiods. In addition, groups were raised entirely under short-day photoperiods or entirely under long-day photoperiods as a control, so that all life stages were exposed to either short or long days. All trials were conducted at $21{ }^{\circ} \mathrm{C}$ with $75 \%$ relative humidity. Adult mosquitoes from the $\mathrm{F}_{2}$ generation were force-mated [10], offered a human blood meal via a volunteer inserting their arm in their cages, and the eggs were collected upon seed germination paper (Anchor Paper Company, Saint Paul, MN, USA). After 14 days, 200 eggs randomly selected from the $\mathrm{F}_{3}$ cohorts were exposed to a hatching stimulus of $0.5 \mathrm{~g}$ of nutrient broth dissolved in $250 \mathrm{~mL}$ of deionized water for $24 \mathrm{~h}$. Following the first hatch attempt, the oviposition papers were allowed to dry for another $24 \mathrm{~h}$ at a $8 \mathrm{~L}: 16 \mathrm{D}$ short-day photoperiod and 
were then subjected to a second hatch attempt. Upon completion of the second hatch attempt, we chemically cleared all unhatched eggs using acetic acid and sodium chlorite solution. Eggs were considered embryonated if the pharate first instar larvae eye spots, egg burster, and hatching spine were easily noticeable. Five replicates of $\mathrm{F}_{3}$ eggs per $\mathrm{F}_{2}$ life stage subjected to diapause conditions were used with the exception of the $\mathrm{F}_{2}$ egg treatment. Percent of viable eggs was calculated as: (number of larvae hatched + number of embryonated eggs)/(number of larvae hatched + number of embryonated eggs+ non-embryonated eggs). Percent diapause was calculated as: (no. of embryonated eggs not hatching after two hatching attempts/no. of viable eggs).

Aedes $j$. japonicus prediapause and viability data were analyzed using Chi-square followed by pairwise comparisons of percent hatch for different life stages by Fisher's exact test using Prism 7 for Mac OSX (GraphPad Software, Inc., 2017, La Jolla, CA, USA).

The evolution of diapause in Aedes was assessed by testing for phylogenetic signal in known diapause states and by ancestral state reconstruction of those states across the phylogeny of the Aedini from Soghigian et al. 2017 [11]. This phylogeny was trimmed with the R function drop.tip from the package ape [12] to taxa for which diapause states had been experimentally determined (Table S1). Pagel's $\lambda$ [13], a measure of phylogenetic signal, was estimated for these states given the Aedini phylogeny with fitDiscrete in the R package geiger [14]. The resulting model from fitDiscrete was compared to a model assuming no phylogenetic signal $(\lambda=0)$ with a likelihood ratio test approximated by a chi-squared distribution in R; a significant difference between these models thus would indicate a better fit of the model with Pagel's $\lambda$ and provide evidence of phylogenetic signal in diapause state.

Next, ancestral states were evaluated across nodes of the phylogeny by generating 1000 stochastic character maps with make.simmap from the R package phytools [15] under a model in which transitions between diapause states had an equal rate (model = "ER" in make.simmap), the best fitting model based on corrected Akaike information criterion (AICcs). The posterior probabilities were summarized at nodes to infer the best supported ancestral state for each node and visualized these posterior probabilities as pie charts on the trimmed phylogeny. Where different populations of the same species exhibit different diapause states, an equal probability of that diapause condition was applied for a given species. For Aedes vexans, where some populations exhibit facultative diapause but the prediapause condition in terms of maternal or embryonic controls is unknown, equal probabilities for either diapause condition were used in the inference of ancestral states.

\section{Results}

The life stage of a mosquito exposed to short-day photoperiods (8 L:16 D) had a significant effect of the hatch rate of $\mathrm{F}_{3}$ eggs $\left(\chi^{2}, p<0.01\right)$ (Table 1 and Figure 2). The exposure of the maternal adult $\mathrm{F}_{2}$ generation to short days resulted in $\mathrm{F}_{3}$ egg diapause (no hatch) irrespective of all other $\mathrm{F}_{2}$ life stage treatments. The hatch rate of eggs produced from egg- and larval-stage exposure to short-day photoperiods was not different from the controls that had not been exposed to any short-day photoperiods (Fisher's exact test, $p<0.01$ ). Short-day treatments on $\mathrm{F}_{2}$ pupae elicited a weak diapause response, resulting in a hatch rate that was lower than that of the controls but higher that of the adults that had been exposed to short days (Fisher's exact test, $p<0.01$ ). All larvae maintained at a short-day photoperiod continued development to pupal ecdysis. 
Table 1. Effect of a short-day photoperiod (8 L:16 D) on Aedes japonicus japonicus $\mathrm{F}_{2}$ life stages on $\mathrm{F}_{3}$ percent diapause. All other $\mathrm{F}_{2}$ life-cycle stages were exposed to a long-day photoperiod (14 L:8 D). * = eggs of the $\mathrm{F}_{2}$ generation were tested for percent diapause. Percentages followed by different letters are significantly different (Fisher's exact test, $p \leq 0.01$ ).

\begin{tabular}{ccc}
\hline Life-Cycle Stage & $\boldsymbol{n}$ Eggs & \% Diapause \\
\hline All & 521 & $99.81 \mathrm{~A}$ \\
Adults & 532 & $100 \mathrm{~A}$ \\
Eggs & $507^{*}$ & $2.96 \mathrm{~B}$ \\
Larvae & 536 & $4.66 \mathrm{~B}$ \\
Pupae & 586 & $20.82 \mathrm{C}$ \\
None & 568 & $2.82 \mathrm{~B}$ \\
\hline
\end{tabular}

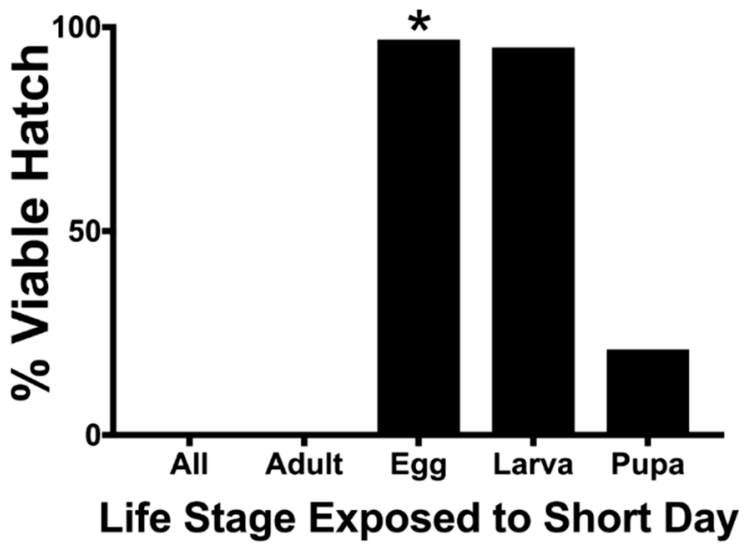

Figure 2. Effect of a short-day photoperiod (8 L:16 D) on Ae. j. japonicus $\mathrm{F}_{2}$ life stages on $\mathrm{F}_{3}$ percent hatch. All other $\mathrm{F}_{2}$ life-cycle stages were exposed to a long-day photoperiod (14 L:8 D). * = eggs of the $\mathrm{F}_{2}$ generation were tested for percent diapause.

Percent egg viability from force-mated pairs of Ae. j. japonicus ranged from 50.7 to $58.6 \%$ with a total mean of $54.2 \%, 95 \%$ CI $(52.9 ; 55.4)$ (Table 2$)$ and were not statistically different (Fisher's exact test $p>0.05)$.

Table 2. Percent viability of force-mated Ae. j. japonicus eggs by life stage exposed to a short-day photoperiod (8 L:16 D). Viability was determined as bleached eggs that display noticeable eye spots and egg bursters. Ae. j. japonicus males and females were force mated according to Gerberg et al. 1994 [10].

\begin{tabular}{cc}
\hline Life Stage Exposed to 8 L:16 D & \% Viability of Eggs \\
\hline ALL & 52.1 \\
Adult & 53.2 \\
Pupa & 58.6 \\
Larva & 53.6 \\
Egg & 50.7 \\
None & 56.8 \\
\hline
\end{tabular}

There was significant evidence of a phylogenetic signal in the prediapause state as the model estimating $\lambda$ had a significantly better fit than the model with no phylogenetic signal (estimated $\lambda=1$, LR $p<0.001)$. Groupings of similar prediapause phenotypes are noticeable in closely related species; however, they were not noticeable in all species (Figure 3). Maternal prediapause is evident in distantly related species, while embryonic prediapause appears in several clusters of closely related species. 


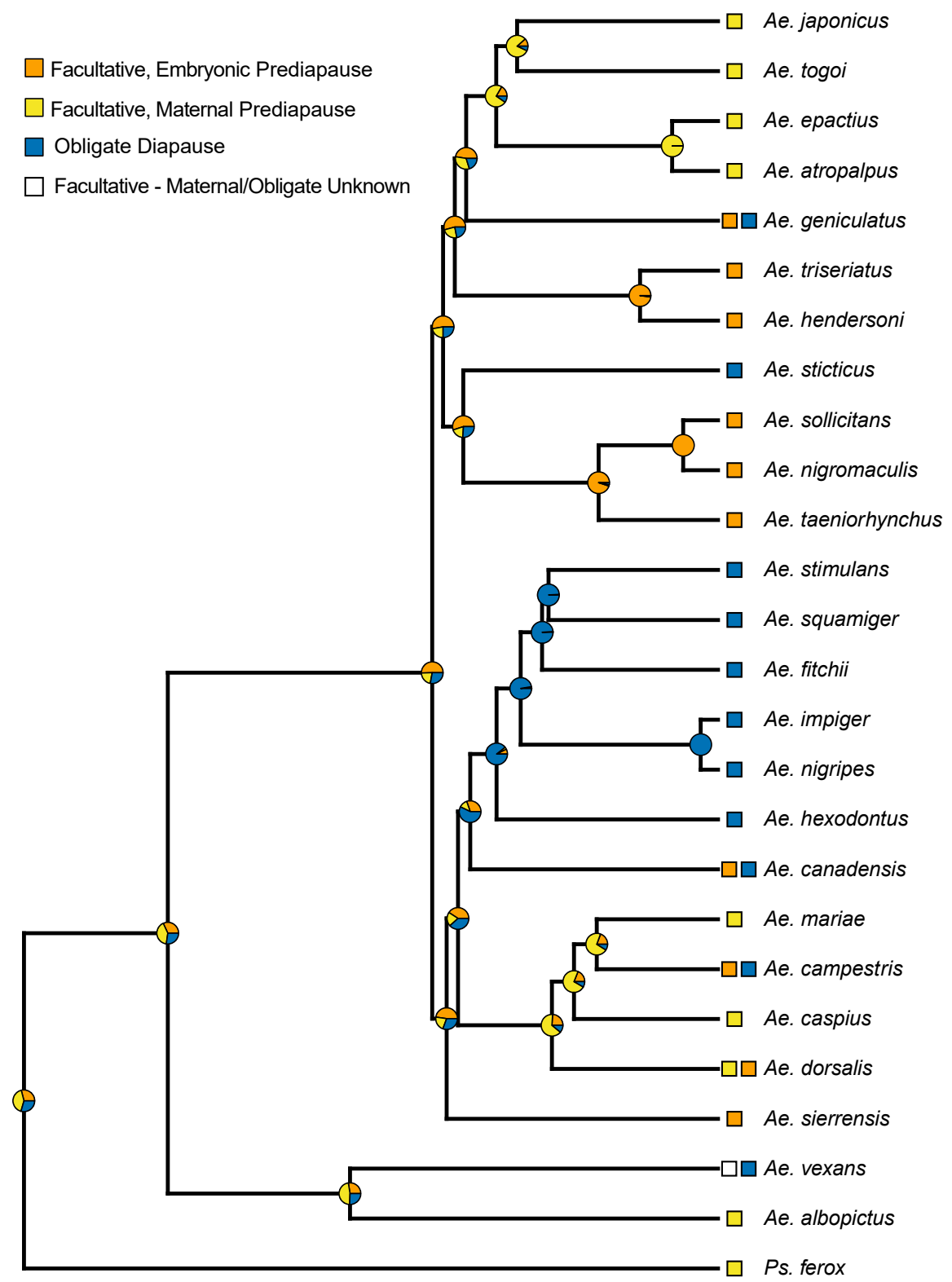

Figure 3. Phylogeny of known prediapause stages in Aedes that undergo embryonic diapause. Species with two prediapause stages have obligate univoltine and facultative multivoltine individuals. Some species utilize multiple life-cycle diapause stages and represented here is the prediapause stage solely to induce embryonic diapause. Phylogeny from Soghigian et al. [11], with posterior probabilities from an ancestral state reconstruction shown on nodes.

Prediapause Definitions:

Facultative Embryonic: The embryo is sensitive to environmental cues needed to induce diapause and is in the overwintering diapause stage.

Facultative Maternal: The $F_{n}$ maternal female is sensitive to environmental cues needed to induce diapause (Figure 1), while the $F_{n+1}$ embryos enter diapause.

Obligate: These mosquitoes lay eggs that enter into an obligate aestivation to avoid dry seasons, or these mosquitoes lay eggs that enter into an obligate diapause to avoid harsh low temperatures and are located more poleward.

Facultative-Maternal/Obligate Unknown: Aedes vexans exhibits an obligate diapause but facultative diapausing populations have been identified; however, the mechanism behind the facultative diapause remains uncertain. 


\section{Discussion}

Aedes $j$. japonicus undergoes embryonic diapause facilitated by the prediapause maternal generation that begins detecting environmental cues in the pupal life-cycle stage and extends into the adult life-cycle stage (Table 1 and Figure 2). This maternal prediapause stage is similar in the closely related Aedes togoi [16], Aedes epactius, and Aedes atropalpus [17] and the more distantly related Aedes albopictus [18] (Figure 3). Low percentages of viable eggs from force-mated females highlight the challenges of rearing Ae. j. japonicus (Table 2). These percentages were consistent across the experiment. With several identifications of virus-infected mosquitoes from the field, a better understanding of the biology of this mosquito is important to understand vector biology and disease transmission [6-8].

Recent molecular phylogenetic analyses have made it possible to begin to examine the evolution of the prediapause stages of Aedes mosquitoes that undergo hibernal, embryonic diapause. Embryonic diapause also occurs in Psorophora ferox and Anopheles walker [19,20]. These non-Aedes species both employ the maternal prediapause stage to induce embryonic diapause in the following generation. Our ancestral state reconstruction suggests a slightly higher probability relative to other possible states that the ancestral diapause state in the Aedini is maternally controlled, diapausing eggs (See Supplemental Figure S1). Due to how little is known about the prediapause condition in the majority of Aedes species, our ancestral state reconstruction, and the conclusions from it, should be considered preliminary until further research on diapause states in additional species can be performed and evaluated within an evolutionary context. That said, beyond the higher posterior probability at basal nodes for a maternal prediapause stage, an ancestral state of facultative maternal prediapuse in the Aedini is consistent with the fact the basal lineage of the Aedini is Psorophora, and many Psorophora species have been described as multivoltine and overwintering in the egg [21]. Multivoltine species with such an overwintering strategy must have facultative diapause, but Psorophora ferox is the only species to our knowledge within Psorophora where this facultative diapause has been further tested to reveal a maternal pre-diapause condition. Thus, our results are consistent with an ancestral state for the Aedini that involves a prediapause stage that is maternally controlled $[2,11]$.

Our results suggest that the evolution of an obligate diapause condition may be preceded by the evolution of facultative embryonic prediapause. All species exhibiting an obligate diapause state, other than Aedes vexans, have an ancestral node with a high probability of embryonic prediapause, or themselves may exhibit embryonic prediapause in some populations. As with considerations for the ancestral condition of prediapause in the Aedini, this observation is highly preliminary and warrants further consideration as additional studies are conducted on diapause.

In Aedes campestris [22], Aedes geniculatis [23], and Aedes canadensis [20], all species have individuals that undergo obligate embryonic diapause to survive northern winters and are univoltine, but more southern populations have individuals capable of facultative embryonic diapause and are multivoltine. While more distantly related, this strategy appears to be convergent, and may indicate that diapause behaviors could be variable across populations of other species, as well. This variability could be a strategy triggered by a warming climate. The studies on the facultative diapause populations of these species date back several decades and merit more current scrutiny along with other poleward species.

\section{Conclusions}

With the addition of identifying the maternal prediapause stage of Ae. j. japonicus and subsequent phylogenetic analysis, we add basic knowledge to the US invasive Ae. j. japonicus, and we present a more detailed understanding of the evolution of embryonic diapause in Aedes mosquitoes. Our preliminary analysis indicates that embryonic prediapause precedes obligate diapause and the ancestral diapause state of Aedini is maternally controlled. Further and more recent sampling of diapause and prediapause states are needed to better understand this fascinating system. 
Supplementary Materials: The following are available online at http://www.mdpi.com/2075-4450/10/8/222/s1, Table S1: Description of species, prediapause stage to induce embryonic diapause, and references; Figure S1: Posterior probabilities of nodes for Figure 3.

Author Contributions: Conceptualization, J.B. and S.P.; Methodology, J.B., J.S., and S.P.; Software, S.P.; Validation, J.B. and S.P.; Formal Analysis, J.B., J.S., and S.P.; Investigation, J.B. and J.S.; Resources, S.P.; Data Curation, J.B.; Writing-Original Draft Preparation, J.B.; Writing-Review and Editing, J.B., J.S., and S.P.; Visualization, J.B. and J.S.; Supervision, S.P.; Project Administration, J.B. and S.P.; Funding Acquisition, S.P.

Funding: J.S. was funded by National Science Foundation Division of Environmental Biology Award \#1754376.

Conflicts of Interest: The authors declare no conflict of interest.

\section{References}

1. Denlinger, D.L.; Armbruster, P.A. Mosquito diapause. Ann. Rev. Entomol. 2014, 59, 73-93. [CrossRef] [PubMed]

2. Denlinger, D.L.; Armbruster, P.A. Molecular physiology of mosquito diapause. Adv. Insect Phys. 2016, 51, 329-361.

3. Diniz, D.F.A.; de Albuquerque, C.M.R.; Oliva, L.O.; de Melo-Santos, M.A.V.; Ayres, C.F.J. Diapause and quiescence: Dormancy mechanisms that contribute to the geographical expansion of mosquitoes and their evolutionary success. Parasite Vectors 2017, 10, 310. [CrossRef] [PubMed]

4. Kaufman, M.G.; Fonseca, D.M. Invasion biology of Aedes japonicus japonicus (Diptera: Culicidae). Ann. Rev. Entomol. 2014, 59, 31-49. [CrossRef] [PubMed]

5. Andreadis, T.; Anderson, J.; Munstermann, L.; Wolfe, R.; Florin, D. Discovery, distribution and abundance of the newly introduced mosquito Ochlerotatus japonicus (Diptera: Culicidae) in Connecticut, USA. J. Med. Entomol. 2001, 38, 774-779. [CrossRef] [PubMed]

6. Westby, K.M.; Fritzen, C.; Paulson, D.; Poindexter, S.; Moncayo, A.C. La Crosse Encephalitis virus infection in field-collected Aedes albopictus, Aedes japonicus, and Aedes triseriatus in Tennessee. J. Am. Mosq. Control Assoc. 2015, 31, 233-242. [CrossRef]

7. Harris, M.C.; Dotseth, E.J.; Jackson, B.T.; Zink, S.D.; Marek, P.E.; Kramer, L.D.; Paulson, S.L.; Hawley, D.M. La Crosse virus in Aedes japonicus japonicus mosquitoes in the Appalachian Region, United States. Emerg. Infect. Dis. 2015, 21, 646. [CrossRef]

8. Yang, F.; Chan, K.; Marek, P.E.; Armstrong, P.M.; Liu, P.; Bova, J.E.; Bernick, J.N.; McMillan, B.E.; Weidlich, B.G.; Paulson, S.L. Cache Valley Virus in Aedes japonicus japonicus Mosquitoes, Appalachian Region, United States. Emerg. Infect. Dis. 2018, 24, 553. [CrossRef]

9. Kappus, K.D.; Venard, C.E. The effects of photoperiod and temperature on the induction of diapause in Aedes triseriatus Say. J. Insect Physiol. 1967, 13, 1007-1019. [CrossRef]

10. Gerberg, E.J.; Barnard, D.; Ward, R. Manual for Mosquito Rearing and Experimental Techniques; American Mosquito Control Association, Inc.: Lake Charles, LA, USA, 1994; p. 98.

11. Soghigian, J.; Andreadis, T.G.; Livdahl, T.P. From ground pools to treeholes: Convergent evolution of habitat and phenotype in Aedes mosquitoes. BMC Evol. Biol. 2017, 17, 262. [CrossRef]

12. Paradis, E.; Claude, J.; Strimmer, K. APE: Analyses of Phyl/Bioinformatics/btg 412. Bioinformatics 2004, 20, 289-290. [CrossRef] [PubMed]

13. Pagel, M. Inferring the historical patterns of biological evolution. Nature 1999, 401, 877-884. [CrossRef] [PubMed]

14. Harmon, L.J.; Weir, J.T.; Brock, C.D.; Glor, R.E.; Challenger, W. GEIGER: Investigating evolutionary radiations. Bioinformatics 2008, 24, 129-131. [CrossRef] [PubMed]

15. Revell, L.J. phytools: An R package for phylogenetic comparative biology ogenetics and Evolution in R language. Methods Ecol. Evol. 2012, 3, 217-223. [CrossRef]

16. Galka, B.E.; Brust, R.A. The effect of temperature and photoperiod on the induction of larval diapause in the mosquito Aedes togoi (Theobald) (Diptera: Culicidae). Can. J. Zool. 1987, 65, 2262-2265. [CrossRef]

17. Anderson, J. Influence of photoperiod and temperature on the induction of diapause in Aedes atropalpus (Diptera, Culicidae). Entomol. Exp. Appl. 1968, 11, 321-330. [CrossRef]

18. Mori, A.; Oda, T.; Wada, Y. Studies on the egg diapause and overwintering of Aedes albopictus in Nagasaki. J. Trop. Med. 1981, 23, 79-90. 
19. Hurlbut, H.S. Further notes on the overwintering of the eggs of Anopheles walkeri with a description of the eggs. J. Parasitol. 1938, 24, 521-526. [CrossRef]

20. Pinger, R.R.; Eldridge, B.F. The effect of photoperiod on diapause induction in Aedes canadensis and Psorophora ferox (Diptera: Culicidae). Ann. Entomol. Soc. Amer. 1977, 70, 437-441. [CrossRef]

21. Crans, W. A classification system for mosquito life cycles: Life cycle types for mosquitoes of the northeastern United States. J. Vector Ecol. 2004, 29, 1-10.

22. Tauthong, P.; Brust, R.A. The effect of photoperiod on diapause induction, and temperature on diapause termination in embryos of Aedes campestris Dyar and Knab (Diptera: Culicidae). Can. J. Zool. 1977, 55, 129-134. [CrossRef] [PubMed]

23. Sims, S.R.M.; Munstermann, L.E. Egg and larval diapause in two populations of Aedes geniculatus (Diptera: Culicidae). J. Med. Entomol. 1983, 3, 263-267. [CrossRef] [PubMed]

(C) 2019 by the authors. Licensee MDPI, Basel, Switzerland. This article is an open access article distributed under the terms and conditions of the Creative Commons Attribution (CC BY) license (http://creativecommons.org/licenses/by/4.0/). 